\begin{tabular}{|l|l|}
\hline AV -3 & 1075 \\
\hline
\end{tabular}

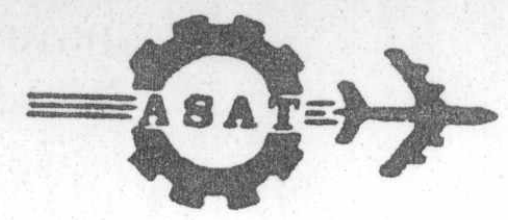

\title{
COMMUNICATION SYSTEM IDENTIFICATION THROUGH ADAPTIVE MODELING OF THE COMMUNICATION CHANNEL
}

BRIG. GEN. DR. NABIL M. ELNADY *

\author{
ENG. EMMANOEL S. HANNA
}

\author{
ABSTRACT
}

In this paper,attempts are made to profile the radio propagation path linking an intercepted transmitter site to the signal collection facility. The main objective is to extract fingerprinting clues to identify the transmitter locality. Different methods of power spectrum estimation techniques are applied and compared w.r.t. their usefulness in identifying the multipath channel. A novel system is proposed to act as a transmitter locality identifier. The system is based on adaptive modeling of the faded channel. The system configuration is presented., the control software is developed and sample run-outs of the developed programs are presented and commented.

* The Chief of Chair of Electronic Warfaree the MTC ,Cairo. ** Ph.D. Aspirant, the MTC,Cairo. 


\section{INTRODUCTI ON:}

Multipath phenomenon [1] has always been very annoying when recelving electromagnetic waves transmitted from a distant locality. The multiplicity of propagation paths, especially when sky wave hops are involved, lead to the well-known fading effect. Fig.1. illustrates the different sky wave paths involved with a single hop communication channel.

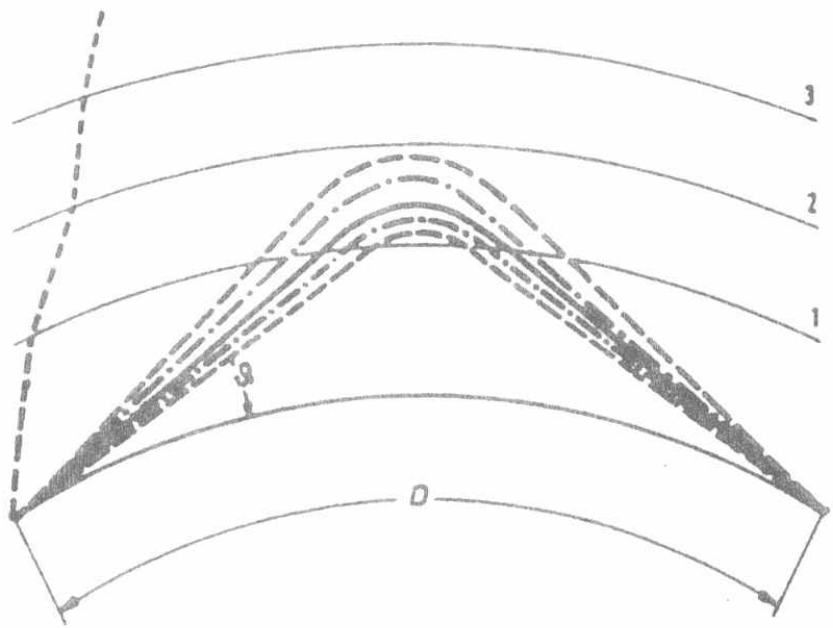

1 Lower effective bounctary of the active ionosheric layer

2 Height of the maximum electron density

3 Upper effective boundary of the active ionospheric layer

- Radiation paths with high and low angles of elevation, freq. much below MUF

-.- Radiation paths with high and low angles of elevation, freq. only a litile below MUF

Radiation path of the MUF

-...- Radiation path of a frequency above the MUF

D Skip distance at the MUF

$\vartheta$ Angle of elevation of the radiation

Fig.1. Diagram of Different sky wave propagation paths[2]

In this paper we will utilize the multipath fading phenomenon to our advantage. We shall use this phenomenon to determine if a recelved signal is originating from the same area. The multipath affecting two independent signals is normally also independent. Thus the rate of fade in the two signal's spectrum will be different. This fact $1 s$ very useful for determining if a complex signal is one signal or if it is actually the sum of two or more independent signals. The procedure for observing the multipath is to perform a frequency raster of the spectrum analyzer display. The equipment configuration for rastering the spectrum is shown in Fig. 2. Two spectrum analyzers were used for this experiment, the HP5866S (Hewlett Packard model) and the SA5004/16 (Scientific Atlanta model). Control software was developed and records of the time history of the signal's spectrum were obtained (see Fig. 3 ). 


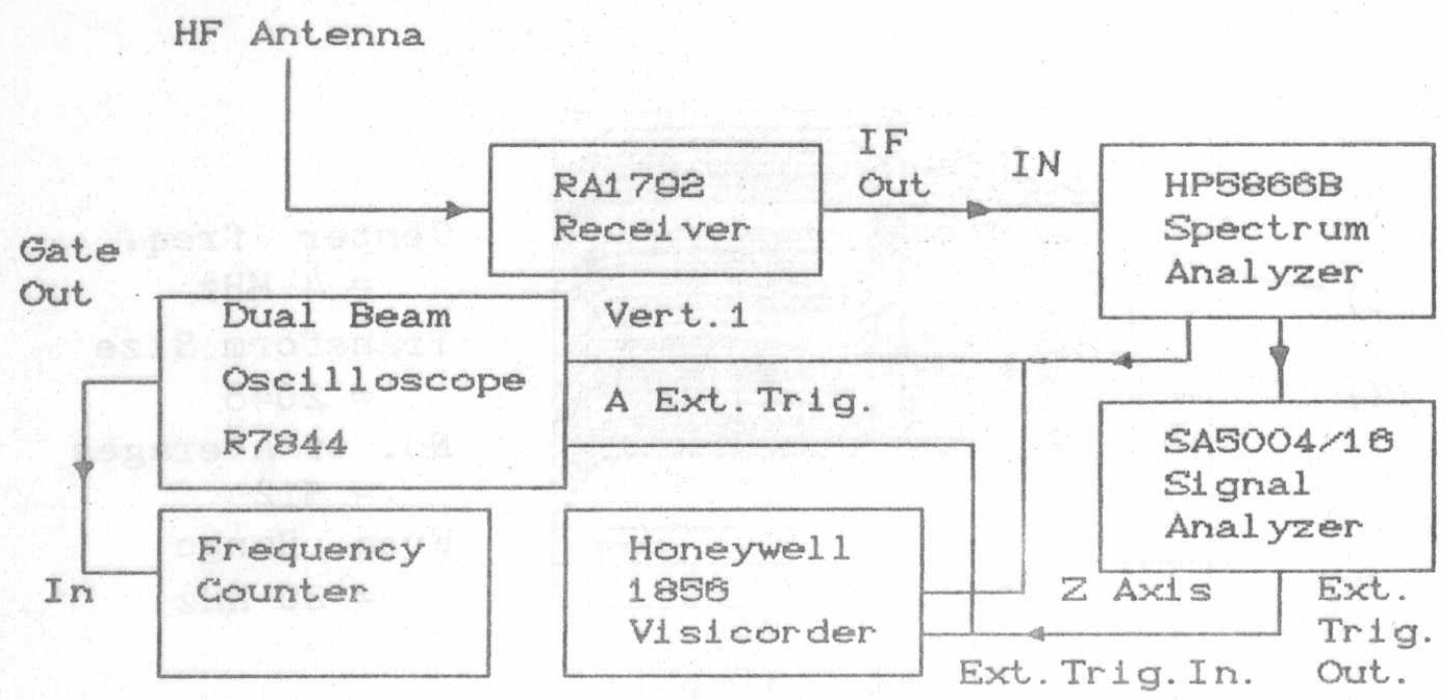

Fig.2. Equi pment Conflguration for Rastering

Multipath fading will create a null in the spectrum. The propagation path that a signal travels over changes with time. This change is usually very slowly varying,taking several seconds to minutes to observe. The effect on the signal's spectrum is to have the fading null change 1 ts position infrequency at different times. Thus a null at one instant of time will be observed to move across a signal's spectrum. Fig. 3 illustrates this phenomenon obtained experimentally $1 \mathrm{n}$ the laboratory using the above mentioned equipment configuration. It is shown in this figure that separate intercepted signals will have two distinctive null patterns representing their unique propagation paths.

\section{DIFFERENT METHODOLOGIES FOR IDENTIFICATION OF THE PROPAGATION PATH :}

In this paper we shall be concerned with the case of unmodulated carrier (with frequency fc). The received signal for the case of discrete multipaths given by

$$
r(t)=\sum_{n} a_{n}(t) e^{-j 2 \pi f_{c} c_{n}(t)}
$$

we expect that the delays $\tau_{n}(t)$ associated with different signal paths to change at different rates and in a random manner. This $i$ mplies that the recelved signal $r(t)$ can be modeled as a random process. When there a large number of paths, the central $1 i \mathrm{mit}$ theorem can be applied. That is, $r(t)$ can be modeled as a complex-valued Gussian random process. This means that the time-variant 1 mpulse response $c(\tau$; $t)$ is a complex-valued Gussian random process the $t$ variable. When the impulse response $c(\tau ; t)$ is modeled as a zero mean complex-valued gusssian process, the envelope $|c(\tau ; t)|$ at any instant(t) is a Rayleigh-distributed [ 2 ] and the channel is said to be a Raylelgh fading channel. In the case where there are fixed signal reflectors in the medium in addition to randomly moving scatterers, $c(\tau ; t)$ can no longer be modeled as having a zero mean. In this case, the envelope $|c(\tau ; t)|$ has a Rice distribution [2] and the channel is said to be a Ricean fading channel. In our analysis we shall consider only the Raylelgh-distributed envelope statistics, a model of ten observed on $\mathrm{HF}$ and is widely accepted. 


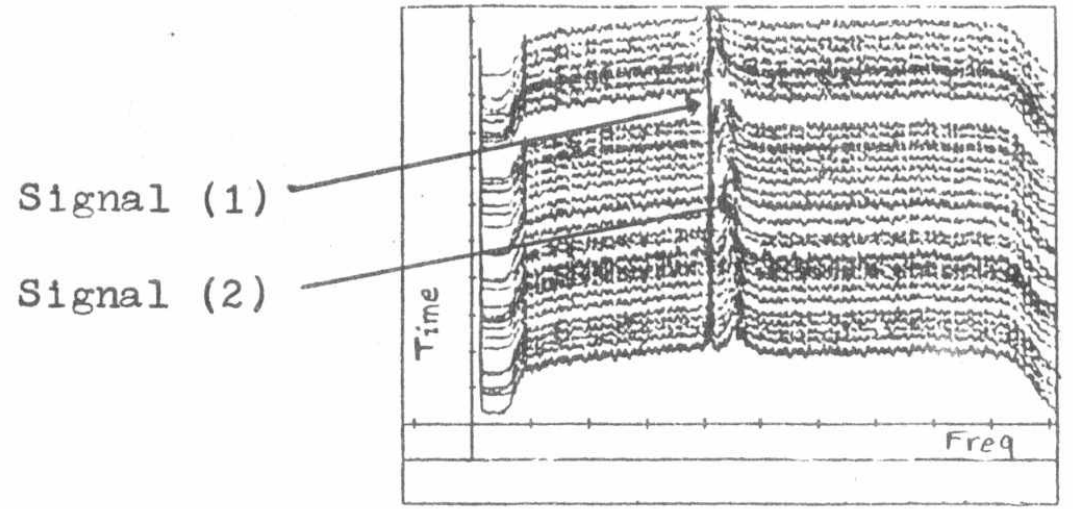

(a) - At 9 a.m.

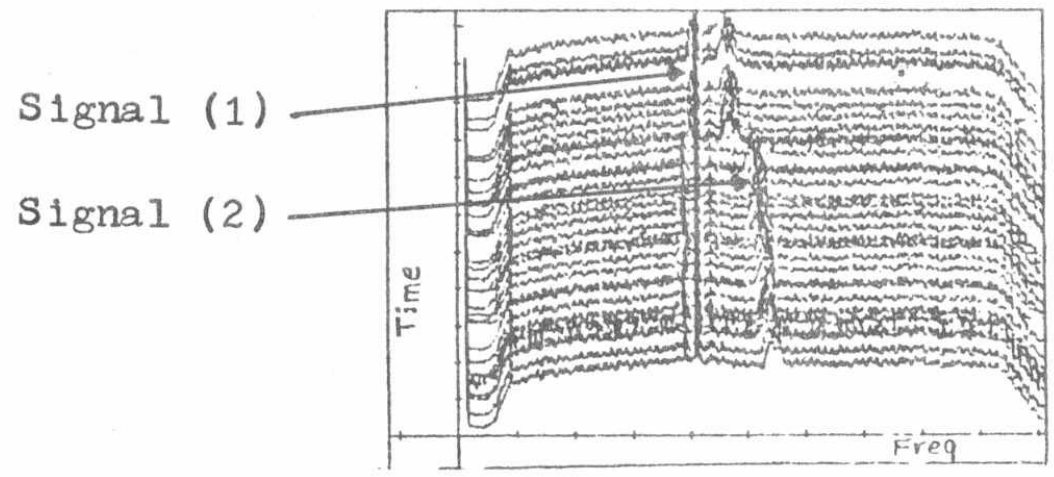

(b) - At $10 \mathrm{a} \cdot \mathrm{m}$.

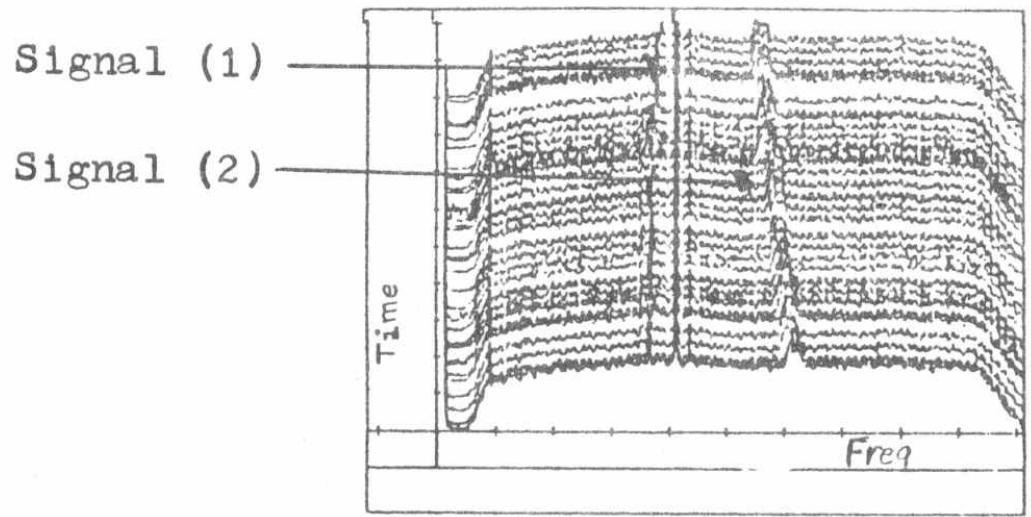

(c) - At 11 a.m.

\section{Center freq. $=4 \mathrm{MHz}$}

Transform Size

$=2048$

No. of Averages

$=512$

Freq. Range

$=20 \mathrm{KHz}$

Same Settings

Same Settings

F1g.3. The Time History of the Intercepted Signal spectrum 
The first methodology that we shall adopt in this paper is to characterize the fading multipath channel by suitable correlation and spectral density functions. Assume $c(\tau ; t)$ to be wide-sense stationary [4], then the autocorrelation function of $c(\tau ; t)$ is

$$
\phi_{c}\left(\tau_{1}, \tau_{2} ; \Delta t\right)=\frac{1}{2} E\left[c^{*}\left(\tau_{1} ; t\right) c\left(\tau_{2} ; t+\Delta t\right)\right]
$$

Most radio transmission media exhibit uncorrelated scattering ; then the scattering at two different delays is uncorrelated. Thus (2) reduces to

$$
\frac{1}{2} E\left[c^{*}\left(\tau_{1} ; t\right) c\left(\tau_{2} ; t+\Delta t\right)\right]=\phi_{c}\left(\tau_{1} ; \Delta t\right) \delta\left(\tau_{1}-\tau_{z}\right)
$$

At $\Delta t=0, \phi(\tau ; 0)$ is the average power output of the channel as a function of the time delay $\tau$. $\phi_{c}(\tau)$ is called the Multipath Intensity Profile or the Delay Power Spectrum of the channel. In practice, the function $\phi_{c}(\tau ; \Delta t)$ is measured by transmitting a wide band signal (or equivalently very narrow pulses) and cross-correlating the received signal with a delayed version of itself. Fig. $4 a$ illustrates $\phi_{c}(\tau) . T_{m} ;(t h e$ range of values of $\tau$ over which $\phi_{c}(\tau)$ is essentially nonzerol is called the Multipath spread of the Channel. An analogous characterization of the time-variant multipath channel can be performed in the frequency domain. $\phi_{c}(\Delta f)$; the autocorrelation function in the frequency variable [4] is given by

$$
\phi_{c}(\Delta f)=\int_{-\infty}^{+\infty} \phi_{c}(\tau) e^{-j 2 \pi \Delta f \tau} d \tau
$$

This relationship is illustrated in Fig. 4 b.

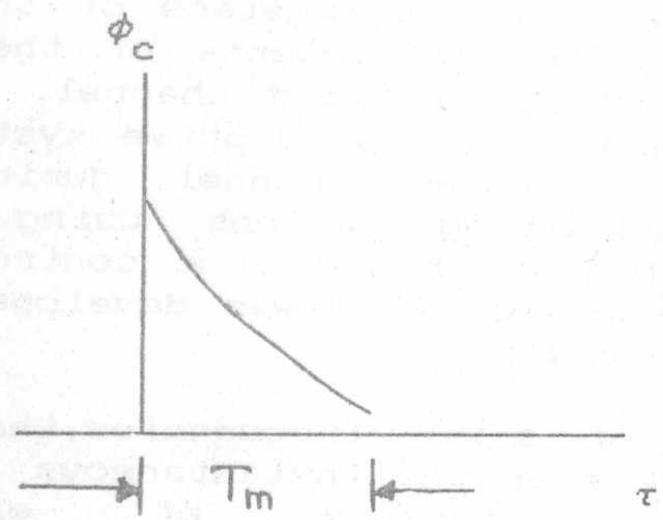

(a)

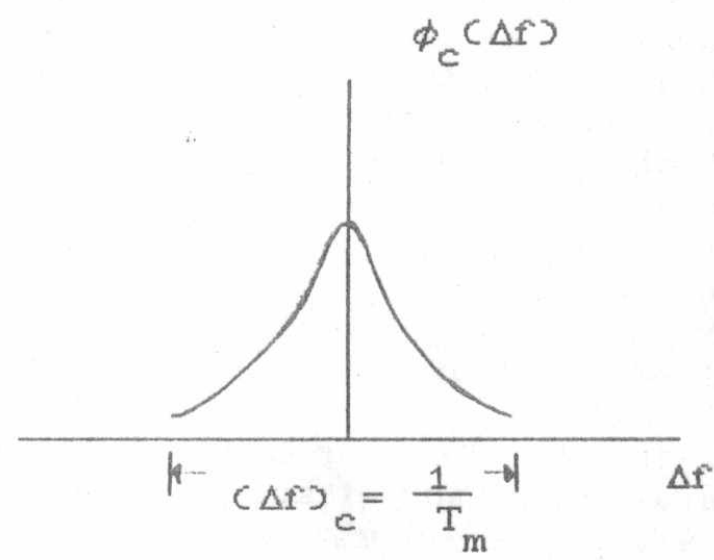

(b) 
$(\Delta f)_{c}$ denotes the coherence Bandwidth Thus two frequencles separated by $>\left(\Delta f_{c}\right.$ are affected differently by the channel. If $(\Delta f)<$ the bandwidth of the transmitted signal, the signal will be severely distorted by the channel. The channel is denoted $1 \mathrm{n}$ this case as a frequency selective channel. Due to the time variations of the channel Doppler shifts and broadening [3] occur. A functioln called the scattering Function of the channel can be characterized [4] and provides a measure of the average power output of the channel as a function of time delay $\tau$ and the Doppler frequency.

\section{APPLYING ADAPTIVE TECHNIQUES}

The second methodology presented here, which is the maln contribution of the paper, is to apply adaptive techniques cwhich are emerging very rapidiy in the field of digital signal processing 2. If the channel characteristics are known and are not varying with time and the signals received are well described and stationary, then the priori knowledge will enable the designer to determine the optimum signal processing method which can be used in the system all the time ce.g.fixed filters, wiener filter - matched filters, etc.). The multipath faded channel cannot be handled in the same manner. Here we apply the princtples of Linear Prediction to charactertze the faded channel and to describe the channel via the LPC Parametrs of the model (see Fig. 5).

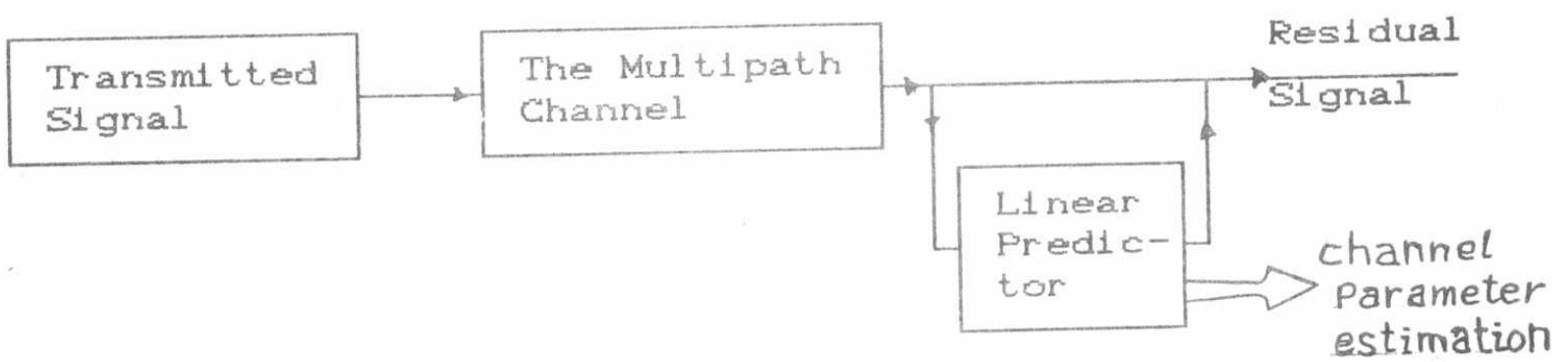

Fig.5. Linear Predicive Coder for the Faded Channel

The linear predictor adapts itself in a mamner such that the residual signal power is minimized. It can be shown that if both the signal and the system are stationary, the parameters of the 1 inear prediction filter are equal to the coefficients of the all-pole filter that is assumed to model the faded channel. A fast adaptation algorithm is needed such that the adaptive system may track the rapid changes in the faded channel during transmission. Intercepting remote broadcasting stations using an equipment configuration similar to that of Fig.2, a control program (to control the HP5866S Spectrum Analyzer) was developed (see Appendix to perform the following tasks:

1-Tuning the analyser to a maximum of seven frequencies.then measuring and displaying the time course of the instantaneous (and eventualy the average) intensity of each signal. The results are displayed in Fig. 6.

2-In order to perform first hand comparison cin respect to the colocality of transmitting stations), the maximum amplitude excursion of the average intensity of each intercepted station 15 


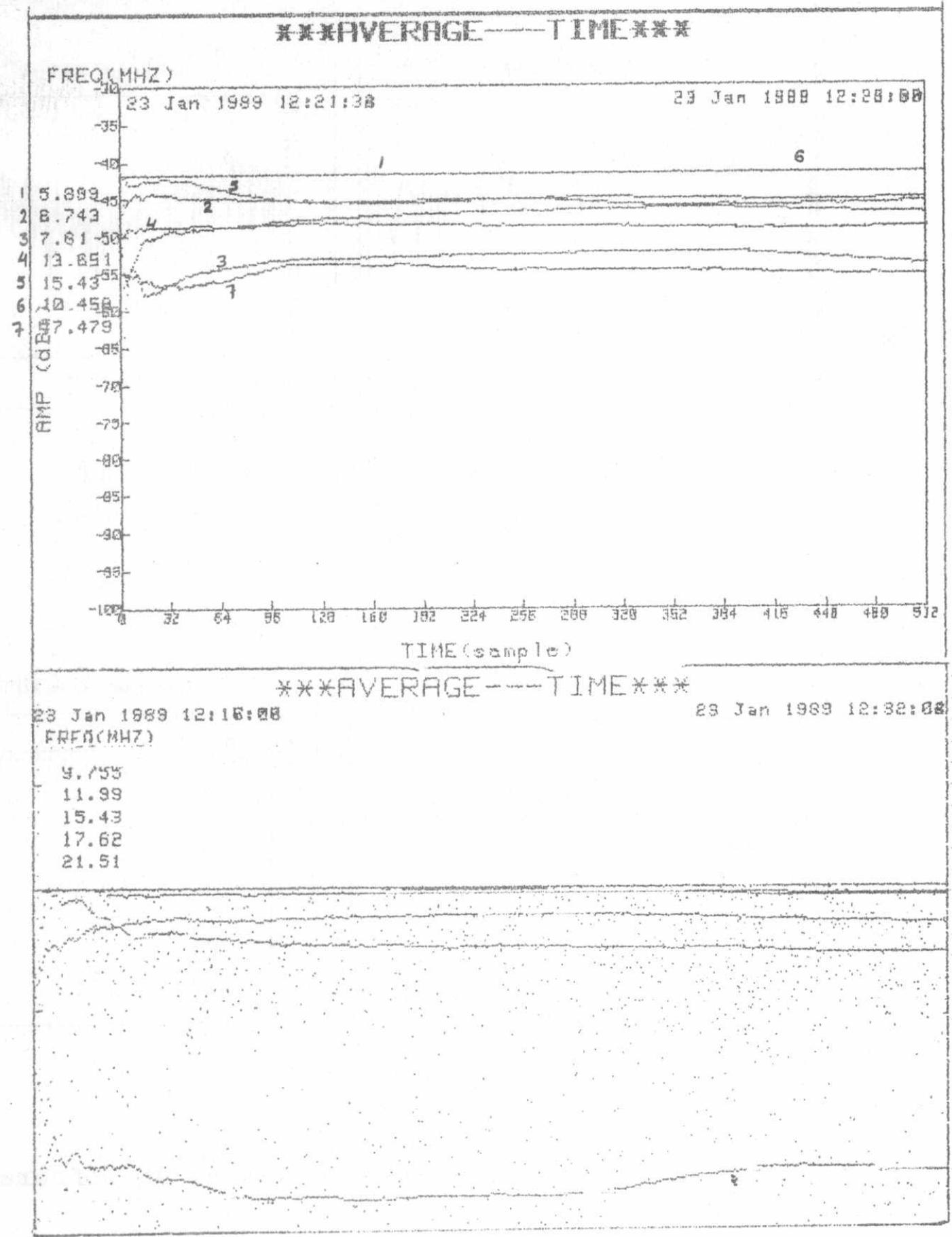


$r$
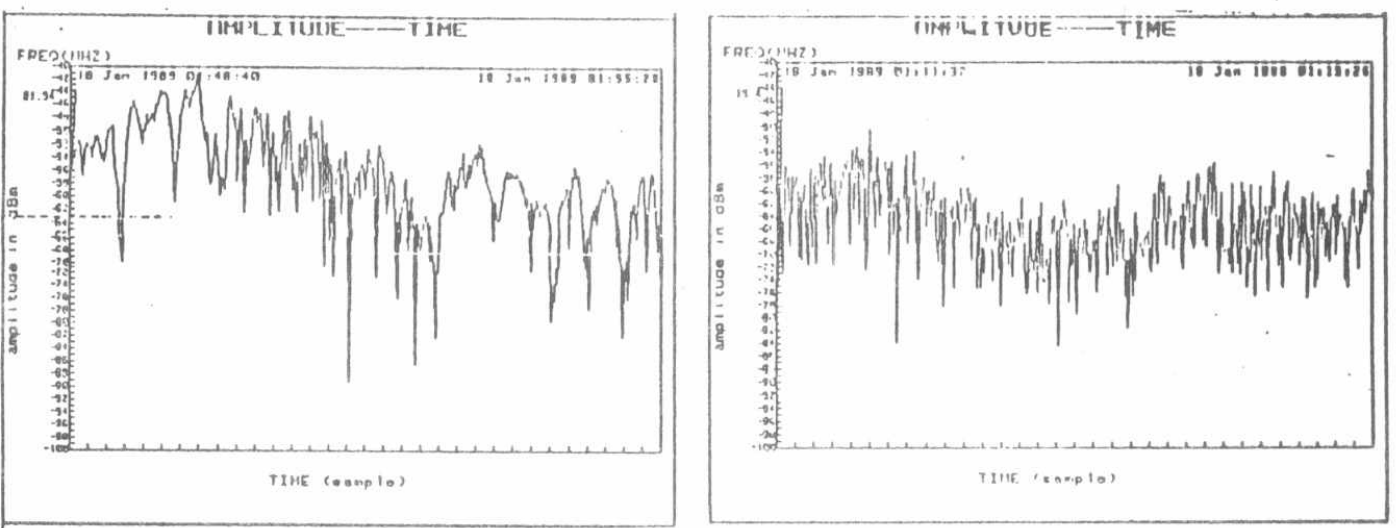

a- Instantaneous amplitude variations of an intercepted signal
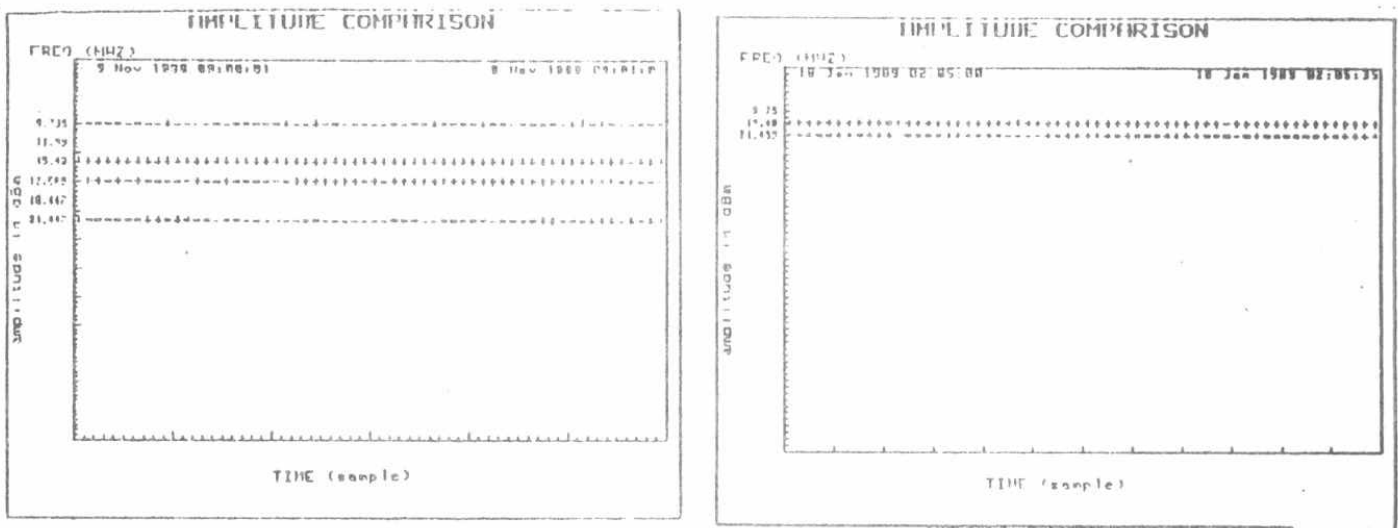

b- Comparative Displays

Fig.7. Qualitative Compar ative Displays of Different Interceptions

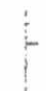



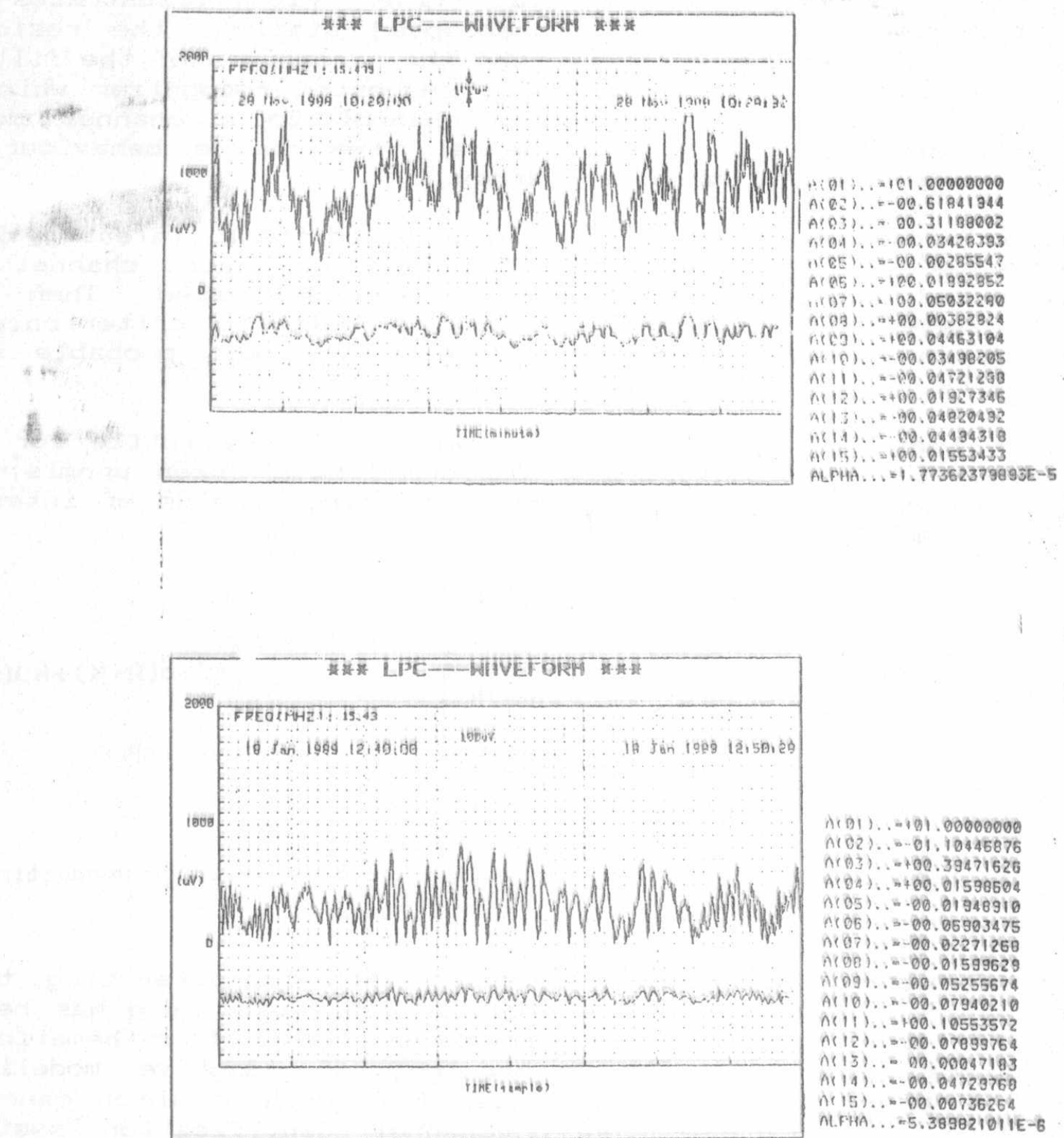

A(D) . . $=181.00000000$ n(c2)... - . 104,1046876 $n(\theta) 3) \ldots=00.39471626$ $A(04), n+00.01598504$ A(05)... - .0.01948910 A(OE).. $3-00.05903475$ $n(07)=-00.02271258$ $A(00),-00.01599629$ ArQ9)... - -00.05255674 $A(10) \ldots n-00.07940210$ $\Lambda(11) \ldots=100.19553572$ A(12), $\rightarrow 00.07899754$ त(13)... $=99.00047183$ $A(14) . .=-00.04723768$ N(15) $=-00.00736254$ AL.FHA. . =5.389821011E-6

Fig.8. Instantaneous amplitude variations, LPC residual sienal and the LPC Filter Coefficients for two distinctive signals. 
then divided into three distinctive levels ccharacterized as ,$+-8=3$. Fig. 7 illustrates the obtained results which may guide in asserting quick recognition.

3-To model the intercepted channel as a 14-section LPC filter and to extract the coefficients of this filter. Fig. 8 illustrates the averaged time course of an intercepted station, the residual signal out of the modeled filter and the parameters of the filter. Fig. 9 illustrates the model for a suggested recognizer which is tasked to compare an intercepted channel to a channel model template. The template of the channel time course behaviour is chosen to be the LPC Parameters Array.

Thus intercepted channels are intercepted, thelr Intensity-Time dependence is monttored, the LPC-Model for each channel is constructed and its LPC-Parameters are evaluated. Then the LPC-Parameters are compared Cusing a suitable criterion,e.g. least squares and the best fit yields the most probable site colocality.

We hope that the ideas presented here will prove futlle for the mentioned tasking. Measurements performed have been promising. The analysis presented is applicable to other fields of 1nterest (e.g. seismology?.

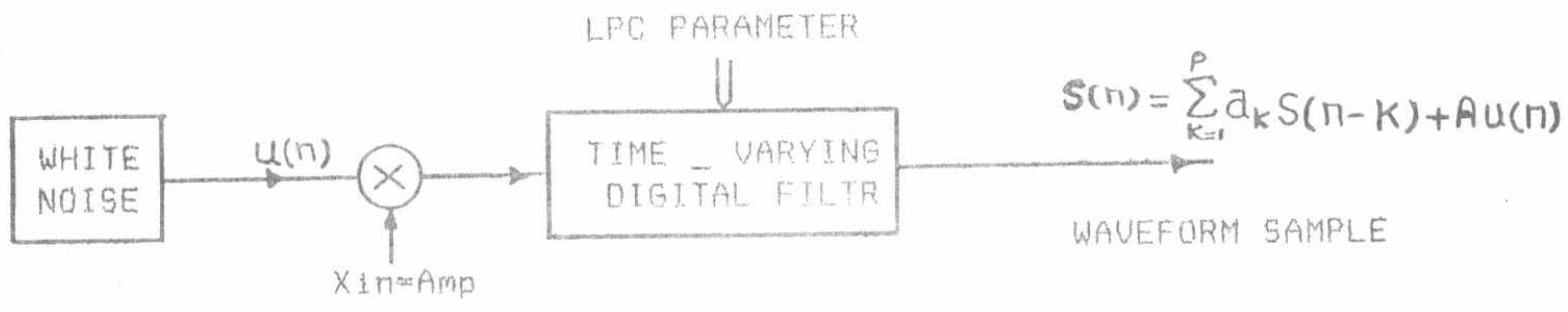

Fig.9 Block diagram of simplified model for waveform productin

\section{CONCLUSI ON}

The first methodology explained deals with characterizing the faded channel by the scattering function. This procedure has been adopted in many situations and involves a lot of mathematical computations. The suggested methodology of adaptive modeling applied to the faded channel promises fast results which can be encompassed in a database-oriented channel identification system

\section{REFERENCES}

1. Treichler.J.R. and Agee,B.G. "A new Approach to Multipath Correction of Constant Modul us Signals", IEEE Trans. on Acous. speech, \&e Signal Process., vol. A.SSP-31, No.2,459-471 (Apr11 1983)

2. Braun, G. "Planning and Engineering of Shortwave Links",J. Wiley and Sons, 39 (1986).

3. Clark, A.P. and McVerry,F., "Channel Estimation for an HF Radio Link "IEE PROC., Vol.128, 33-42 (Feb.1981),

4. Proakis,J.G., "Digital Communications", McGraw-Hill, Inc. ,463 (1983)

\section{NOMENCLATURE}

MUF. . . . . Max Usable Frequency.

$c(\tau ; t)$ : The time-variant 1 mpulse response of the equivalent lowpass channel 


\section{Appendix}

(Sample Flowcharts of the Developed Programs)

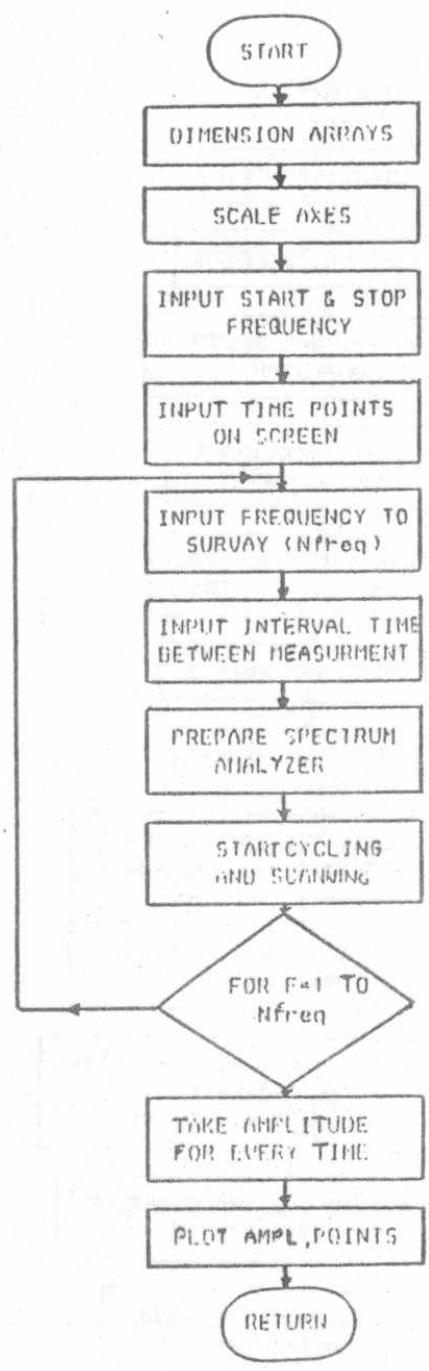

a -

FLON CHLRT FOR WHELLIUDE--.-TIME:

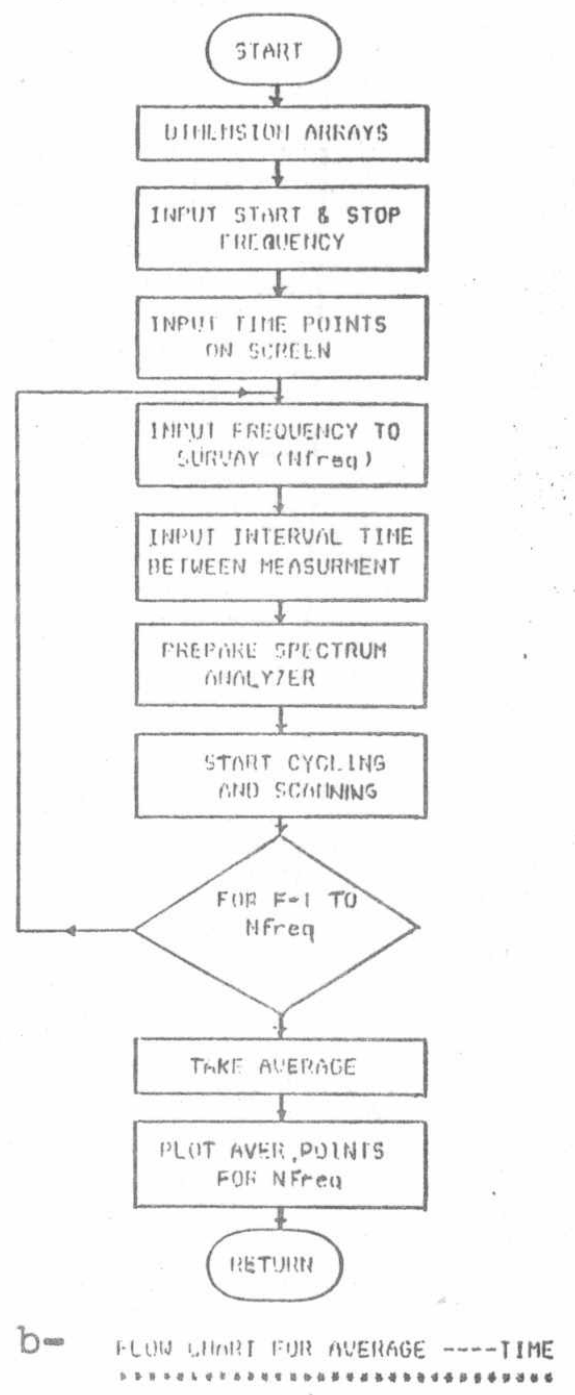




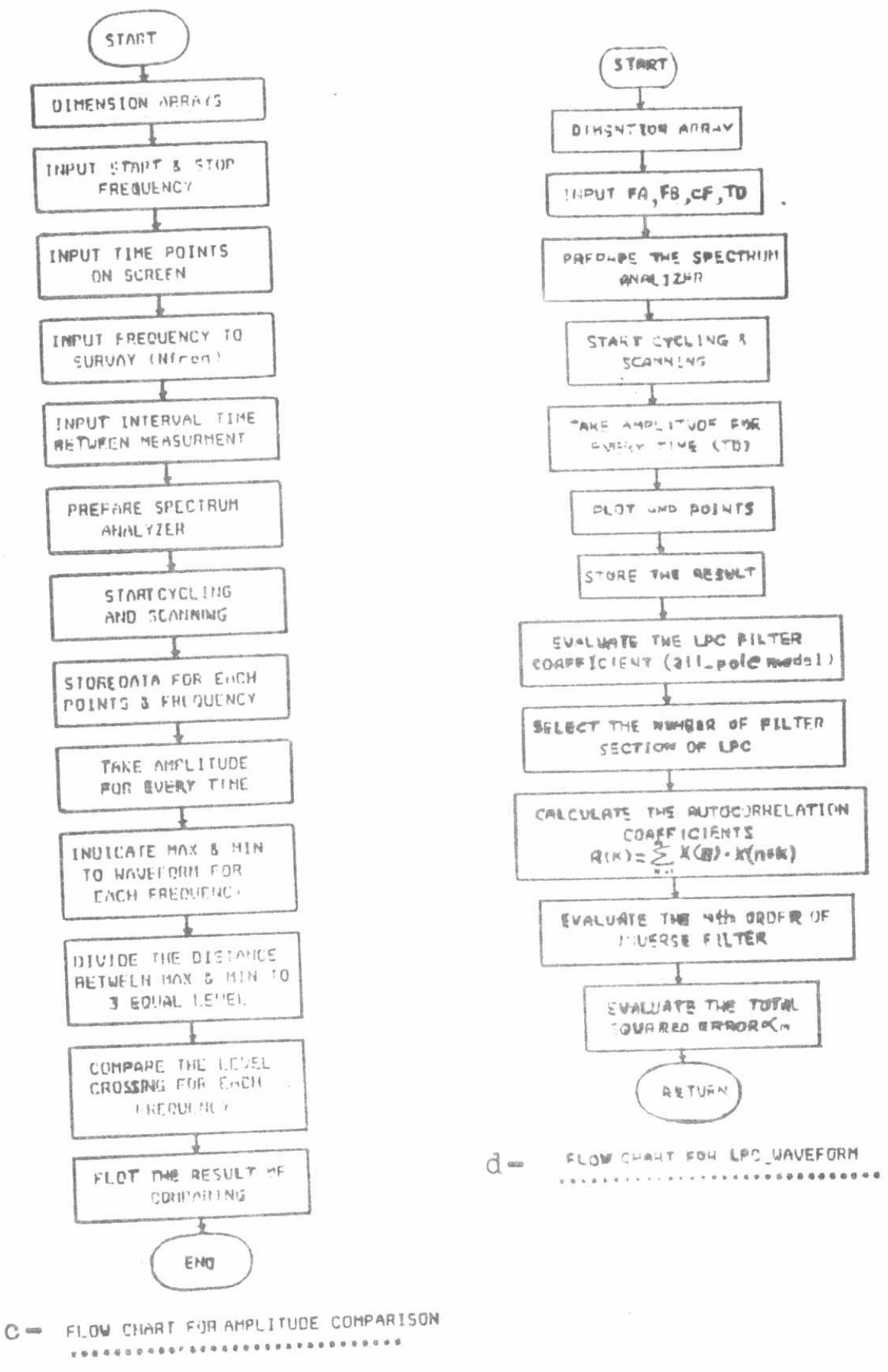




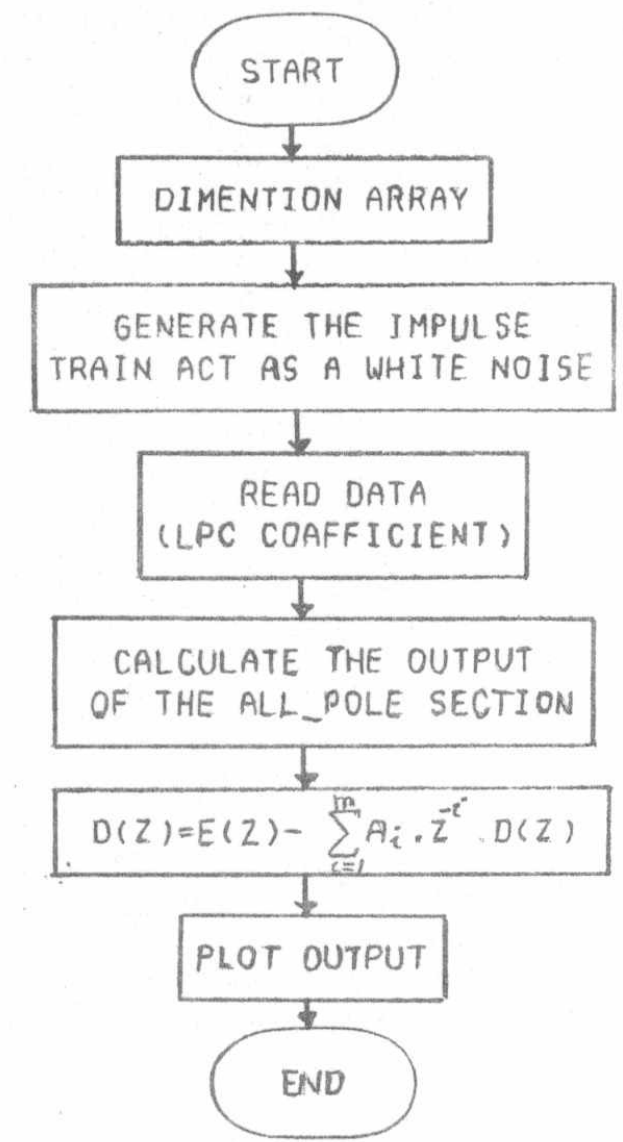

e. FLOW CHART FOR SIMPL IFIEO MODEL FOR WAUEFORM PRODUCTION 